\title{
Breast cancer prevention trials 'can re-start'
}

Washington. A controversial clinical trial of the effectiveness of the drug tamoxifen in preventing breast cancer should be allowed to continue, despite increased estimates of the chances of women who take the drug developing endometrial cancer, the US Food and Drug Administration (FDA) was told last week.

The FDA's oncology advisory committee had been asked to judge whether new estimates of the risk of endometrial cancer meant that the trial should be stopped. It was also asked whether the conditions of eligibility for the trial should be changed.

After much discussion, the panel told the FDA that the trial should continue and that the eligibility criteria should remain unaltered. But it did call for increased monitoring of participants for endometrial cancer, while acknowledging its inability to recommend a screening method, as no one procedure is well enough understood.

The panel said that possible screening methods should be "vigorously evaluated". But it did not discuss the potential dangers of colorectal cancer, even though this may be another possible side-effect of tamoxifen.

The tamoxifen prevention trial is being run by the National Surgical Adjuvant Breast and Bowel Project (NSABP) at the University of Pittsburgh, in Pennsylvania. The aim is recruit 16,000 women at a high risk of developing breast cancer, and randomly assign them either a placebo or tamoxifen for five years. Their progress will be followed for seven years to see whether tamoxifen reduces the number of breast cancers.

Nearly 11,000 women are already taking part in the trial, and last week's meeting should have been the final word on whether or not the trial should continue. But for several reasons the trial has become mired in controversy.

First, the National Cancer Institute (NCI) suspended recruitment of participants at the end of March - along with those in all of the NSABP's other trials - - following the discovery that the quality of data submitted to the NSABP had not been adequately monitored (see Nature 368, 679; 1994).

Most of the NSABP's other trials evaluating treatments for breast and bowel cancer have resumed recruitment of patients. But the NCI is still discussing the conditions under which recruitment to the tamoxifen prevention trial should recommence.

Few doubt that recruitment will be completed, as the National Cancer Advisory Board has already said that the trials should be reopened as soon as possible. Donald Trump, the interim executive director of the NSABP, also says that the NCI has provisionally accepted proposals for tightening up data monitoring.

But the NCI is walking on eggshells. In addition to the institute's own concerns about

the NSABP, Congressman John Dingell (Democrat, Michigan) was due to hold a hearing this week into the University of Pittsburgh's handling of allegations of scientific misconduct. And Senators Connie Mack (Republican, Florida) and Diane Feinstein (Democrat, California) have written to NCI's director, Samuel Broder, asking to be kept closely informed about the prevention trial.

Complicating matters further is a heated debate in both the United States and Britain about whether tamoxifen should be given to healthy women at all. The rationale for the prevention trial is the acknowledged fact that the drug reduces the appearance of new cancers in a large percentage of women who have already had the disease. But some question whether the potential benefit to a healthy woman of avoiding breast cancer outweighs the

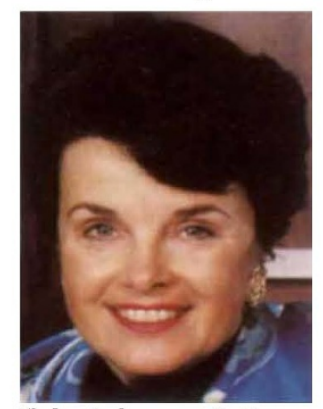

Feinstein: wants to be kept informed.

risks of taking a drug with serious known and suspected side-effects.

Supporters of the trial accept that the women involved should be at higher risk of developing breast cancer than other women of their age group; but they do not agree on what the increased risk should be.

The NSABP's trial recruits women from the age of 35 upwards with the same (or higher) risk of developing breast cancer as a woman of 60 . During the original peer review of the trial, several scientists said that, in view of the risks involved, this 'baseline' risk had been pitched too low.

The prevention trial was approved without modifying the level of risk selected. But last week, Leslie Ford, the NCI's head of the tamoxifen prevention trials, told the FDA that, in practice, the women recruited to the trial were at a much higher risk of developing breast cancer than was called for in the trial protocol.

\section{Japan signs co-operation agreement with UK}

Tokyo. Japan and the United Kingdom this week signed their first formal intergovernmental agreement on science and technology. The agreement is expected to boost the already substantial research links between the two countries.

The agreement was signed by William Waldegrave, the UK minister for public service and science, and Mikio Omi, Japan's minister of state for science and technology, and is a product of a visit to
Craig Henderson, chief of medical oncology at the Mount Zion Medical Center in San Francisco, California, expressed concern at the news. "If the trial shows a benefit, and we recommend tamoxifen as a preventative, it will be given to women with the lower increased risk of breast cancer, [even though] the trial will not have shown the benefit to these women," he said.

Trevor Powles, from the Royal Marsden Hospital in London, gave the FDA panel details of a small trial being carried out to evaluate a promising endometrial screening procedure to catch changes to the endometrium early enough to prevent cancer developing. The women undergoing screening are all post-menopausal, and are members of a pilot tamoxifen prevention trial at the Royal Marsden. Each has at least a 3.8-fold higher risk of contracting breast cancer than other women of the same age.

The possible link between tamoxifen and colorectal cancer is being studied in Britain by the Medical Research Council's Toxicology Unit. The study has been prompted by unpublished findings from a Swedish group, headed by Larferic Rutqvist at the Karolinska Institute in Stockholm suggesting a possible doubling of colorectal cancer among patients taking tamoxifen.

Meanwhile, Britain's Imperial Cancer Research Fund (ICRF) recently began to recruit 15,000 women of 35 and over for a randomized tamoxifen prevention trial. The ICRF is recruiting women whose chances of developing breast cancer are twice as high as other women of the same age - a similar risk level as that in the NSABP's trial. At present, gynaecological examinations will be used to detect endometrial cancer.

The ICRF hopes for 150 fewer cases of breast cancer among the women taking tamoxifen, resulting in 50 fewer deaths. It also anticipates 40 extra cases of endometrial cancer among those taking the drug, with six extra deaths. But the Medical Research Council still insists that it will fund a trial only in women over 40 with a four-fold higher risk of developing breast cancer than other women their own age.

Helen Gavaghan
Japan last year by Waldegrave and Prime Minister John Major (see Nature 365, 480; 1993).

It establishes a formal framework for regular high level meetings to encourage and steer collaborative research, and is partly a response to moves by other advanced nations - such as the United States, Germany and France - which have established bilateral agreements with Japan in recent years. David Swinbanks 